\title{
Innovations and practical applications of intelligent systems in ambient intelligence and humanized computing
}

\author{
David Camacho $^{1} \cdot$ Paulo Novais $^{2}$
}

Published online: 4 February 2017

(C) Springer-Verlag Berlin Heidelberg 2017

\section{Introduction}

Ambient intelligence (AmI) goal is increasing the quality of life and security of the users by introducing the technology into everyday environment, relieving the users' burden in performing normal activities. Therefore, the AmI can be seen as the provision of services in the environment, with a very particular nuance: that these services must be personalized, i.e., tailored to the user's preferences and needs. We consistently find that the behaviour of people can effectively be used as an interaction medium with technology, i.e., it is possible to develop AmI systems that are responsive to people's behaviours (Aztiria et al. 2010), to model their behaviours (Rodríguez-Fernández et al. 2016, 2017) or even to predict their future necessities.

Intelligent Systems, along with the AmI, aims to include technology that is more smart, intelligent and also reliable in the environments, allowing better support for humans when interacting with these environments. Current trends on other areas as Big Data analysis (Iqbal et al. 2016), specially focused on Social analysis (Bello-Orgaz et al. 2016) and Information Fusion, are opening new interesting areas of work, from communities detection (Bello-Orgaz et al. 2017; González-Pardo et al. 2017), new algorithms,

David Camacho

david.camacho@uam.es

Paulo Novais

pjon@di.uminho.pt

1 Computer Science Department, Universidad Autónoma de Madrid, Avda. Tomas y Valiente, n11, 28049 Madrid, Spain

2 Departamento de Informática/Centro ALGORITMI, Escola de Engenharia, Universidade do Minho, Campus de Gualtar, 4710-057 Braga, Portugal systems and frameworks implementations (CampilloSanchez et al. 2015), or ambient-assisted living tools (Rashidi and Mihailidis 2013) amongst others. These, and other related areas, will provide new challenges to AmI and Intelligent Systems in the near future.

This special issue on Innovations and Practical applications of Intelligent Systems in Ambient Intelligence and Humanized Computing is devoted to create a multi-disciplinary discussion forum that bring together researchers from the different fields addressed discussing issues in Intelligent Systems topics involved in the Ambient Intelligence environments. A special emphasis is done to the presentation of concrete systems, discussion of implementation and development challenges and sharing of conclusions achieved and relevant results. From previous state of the art, some contributions have been selected in order to take a step forward over different problems and challenges related to AmI.

\subsection{Contributions of this issue}

The paper entitled "Ubiquitous driving and community knowledge" (Silva and Analide 2016) authored by Silva and Analide presented the PHESS Driving platform enables unobtrusive sensorization of driving actions while acquiring data used to the analysis of driving behavior.

In the paper "Direct trust assignment using social reputation and aging" (Buzzanca et al. 2016) authored Marco Buzzanca et al. the exploitation of trust in virtual environments, on-line services, intelligent pervasive environments and many other scenarios still deserve researchers' interest. In this work the authors investigate, particularly, on how to assess the local (direct) trust a node receives from each of his neighbors. 
The paper entitled "Towards improving feature extraction and classification for activity recognition on streaming data" (Yala et al. 2016) authored by Nawel Yala et al. propose and evaluate an extension of a sensor window approach to perform activity recognition in an online/ streaming setting; recognizing activities when a new sensor event is recorded.

The paper entitled "Context acquisition in auditory emotional recognition studies" (Carneiro et al. 2016) authored by Davide Carneiro et al. describes an environment to access auditory emotional recognition based on a mobile application.

The paper entitled "Agent architecture for crowd simulation in indoor environments" (Pax and Pavón 2016) authored by Rafael Pax and Juan Pavón, purpose an agent based simulation tools for quick prototyping in order to validate social models.

The paper entitled "MAS-based self-adaptive architecture for controlling and monitoring cloud platforms" (Prieta et al. 2017) authored by Prieta et al. presents an architecture to model the control and monitoring system of a Cloud Computing platform by using a Virtual Organization of intelligent agents that self-adapt and reorganize according to the needs of the surrounding environment.

Finally, the paper entitled "Smart environment architecture for robust people detection by infrared and visible video fusion" (Castillo et al. 2016) authored by José Carlos Castillo et al. this paper proposes an architecture for people detection which is focused on robustly detection by infrared and visible video fusion in smart environment.

Acknowledgements This special issue has been achieved by a number of fruitful collaborations. We would like to thank the Editor in Chief of the Journal of Ambient Intelligence and Humanized Computing, Prof. Vincenzo Loia, for his kind support and help during the entire process of publication. In this special issue we processing so many papers in a timely manner would have been impossible but for the timely and active cooperation of the reviewers. The Guest Editors take this opportunity to thank them all. Finally, we are most grateful to the authors for their valuable contributions and for their willingness and efforts to improve their papers in accordance with the suggestions and comments from reviewers and editors.
Bello-Orgaz G, Jung JJ, Camacho D (2016) Social big data: recent achievements and new challenges. Inf Fusion 28:45-59

Bello-Orgaz G, Hernandez-Castro J, Camacho D (2017) Detecting discussion communities on vaccination in twitter. Future Gener Comput Syst 66:125-136

Buzzanca M, Carchiolo V, Longheu A, Malgeri M, Mangioni G (2016) Direct trust assignment using social reputation and aging. J Ambient Intell Human Comput. doi:10.1007/ s12652-016-0413-0

Campillo-Sanchez P, Gomez-Sanz JJ (2015) A framework for developing multi-agent systems in ambient intelligence scenarios. In: Proceedings of the 2015 International Conference on Autonomous Agents and Multiagent Systems (AAMAS'15), 1949-1950

Carneiro D, Pinheiro AP, Novais P (2016) Context acquisition in auditory emotional recognition studies. J Ambient Intell Human Comput. doi:10.1007/s12652-016-0391-2

Castillo JC, Fernández-Caballero A, Serrano-Cuerda J, López MT, Martínez-Rodrigo A (2016) Smart environment architecture for robust people detection by infrared and visible video fusion. $\mathrm{J}$ Ambient Intell Human Comput. doi:10.1007/s 12652-016-0429-5

De la Prieta F, Bajo J, Rodríguez S, Corchado JM (2017) MASbased self-adaptive architecture for controlling and monitoring cloud platforms. J Ambient Intell Human Comput. doi:10.1007/ s12652-016-0434-8(in this issue)

Gonzalez-Pardo A, Jung JJ, Camacho D (2017) ACO-based clustering for Ego Network analysis. Future Gener Comput Syst 66:160-170

Iqbal R, Doctor F, Morea B, Mahmuda S, Yousuf U (2016) Big data analytics: computational intelligence techniques and application areas. Int J Inf Manag. doi: 10.1016/j.jinfomgt.2016.05.020(In press)

Pax R, Pavón J (2016) Agent architecture for crowd simulation in indoor environments. J Ambient Intell Human Comput. doi:10.1007/s12652-016-0420-1

Rashidi P, Mihailidis A (2013) A survey on ambient-assisted living tools for older adults. IEEE J Biomed Health Inf 17(3):579-590

Rodríguez-Fernández V, Menéndez HD, Camacho D (2016) Automatic profile generation for UAV operators using a simulationbased training environment. Prog Artif Intell 5(1):37-46

Rodríguez-Fernández V, Menéndez HD, Camacho D (2017) Analyzing temporal performance profiles of UAV operators using time series clustering. Expert Syst Appl 70(15):103-118

Silva F, Analide C (2016) Ubiquitous driving and community knowledge. J Ambient Intell Human Comput. doi:10.1007/ s12652-016-0397-9

Yala N, Fergani B, Fleury A (2016) Towards improving feature extraction and classification for activity recognition on streaming data. J Ambient Intell Human Comput. doi:10.1007/ s12652-016-0412-1

\section{References}

Aztiria A, Izaguirre A, Augusto JC (2010) Learning patterns in ambient intelligence environments: a survey. Artif Intell Rev 34(1):35-51 